\title{
The Study of Assessment of Gestational Age from Fetal Femur Length by Ultrasonography in Western Maharashtra
}

\author{
Dr. Sandhyarani M. Patil*
}

Associate Professor, Department of Anatomy, R.C.S.M. Govt. Medical College, Kolhapur, Maharashtra, India

\author{
\begin{tabular}{ll}
\hline DOI: $10.36348 /$ SIJAP.2019.v02i09.003 & | Received: 07.09.2019| Accepted: $25.09 .2019 \mid$ Published: 28.09 .2019
\end{tabular} \\ *Corresponding author: Dr. Sandhyarani M. Patil
}

\section{Abstract}

Gestational age determination by ultrasonography is very much essential as it gives accurate estimation. The objective of prsent work is to study the role of ultrasonography for estimation of gestational age and to assess of gestational age by ultrasonographic measurement of femur length. The study was carried out in the Department of Anatomy and in the Department of Radiodiagnosis at R.C.S.M.Government Medical College, Kolhapur, Maharashtra. This prospective cross sectional study consists of 150 normal antenatal women. The fetuses of singleton pregnancy with the physical evidence of normal growth without gross structural abnormalities with the gestational age ranging from18-40 weeks were included. The fetal femur length was measured by ulrasonography. The mean values of femur length in $\mathrm{cm}$. along with respective standered deviation (sd) were computed for each gestaional age from 18-40 weeks. The correlation and regression analysis has been carried out to quantify the relationship between gestational age in weeks and femur length in $\mathrm{cm}$. The femur length goes on increasing from 18 weeks to 40 weeks. The diphyseal length of femur is directly related to the gestational age. The simple linear regression equation derived for the calculation of gestational age using diphyseal length of femur. The relationship between the diphyseal length of femur and the gestaional age is linear and direct.Measurements of diphyseal lengths of femur can be an important parameter for estimating gestational age from 1840 weeks of gestation.

Keywords: Femur length, sonography, gestational age.

Copyright @ 2019: This is an open-access article distributed under the terms of the Creative Commons Attribution license which permits unrestricted use, distribution, and reproduction in any medium for non-commercial use (NonCommercial, or CC-BY-NC) provided the original author and source are credited.

\section{INTRODUCTION}

Knowledge of gestational age is critical for obstetric decision making throughout the pregnancy. Gestational age and fetal weight are the two most important determinants of fetal viability and survival. Without the accurate knowledge of gestational age diagnosis of conditions such as prolonged or post term pregnancy and intrauterine grow retardation is often impossible. Appropriate management of preterm labour or a medically complicated pregnancy depends on accurate estimate of fetal age and weight [1].

An average normal gestational age, which is the length of the pregnancy, is approximately 40 weeks, with a normal range of 38-42 weeks. Reliable estimation of gestational age is essential as it allows appropriate scheduling of a womans antenatal care, informs obstetric management decisions and facilitates the correct interpretation of fetal growth assessment [2]. Recent advances in fetal imaging have been result of technological achievements in sonography and magnetic resonance imaging, with dramatic improvements in resolution and image display. A sonographic examination performed with the exacting recommended standerds of American institute of Ultrasound in medicine offers vital information about fetal anatomy, physiology, growth and well being [3]. Limbs are traditionally assessed during pregnancy as markers of fetal growth, nutrition, and gestational age. However evaluation of fetal limbs and identification of abnormalities may also aid in the diagnosis of various chromosomal and non-chromosomal conditions as well as narrow the differential diagnosis in cases where associated abnormalities have also been identified [4].

\footnotetext{
AIM

To measure the femur length of fetus for prediction of gestational age by ultrasonography.

\section{OBJECTIVE}

- To study the role of ultrasonography for estimation of gestational age.
} 
- To study the assessment of gestational age by ultrasonographic measurement of femur length.

\section{MATERIAL AND METHODS}

The study was carried out in the Department of Anatomy and in the Department of Radiodiagnosis at R.C.S.M.Government Medical College, Kolhapur, Maharashtra. This prospective cross sectional study consists of 150 normal antenatal women. The study protocol was approved by the institutional Ethical Committee. The statistical software namely SPSS used for the analysis of the data and Microsoft Word and Excel have been used to generate graphs etc.

\section{Inclusion Criteria}

The fetuses of singleton pregnancy with the physical evidence of normal growth without gross structural abnormalities with the gestational age ranging from18-40 weeks were included. The research subjects were healthy Indian women from district of Kolhapur. Their age ranged from 20-35 years with weight of 40$60 \mathrm{~kg}$ at the time of examination and their height was between 5 feet and 5 feet 6 inches. The subject had a regular menstrual history. All examinations were performed using the same examiner to minimize the measurement errors.

\section{Exclusion Criteria}

The cases of maternal diseases known to affect normal fetal growth such as chronic hypertension, gestational diabetes melitus, anaemia, hypothyroidism etc. were excluded. The pregnancies with multiple gestations or other complications such as fetal growth restriction, pre-eclampsia, pregnancy induced hypertension, placenta previa or major fetal abnormalities were excluded.
The grey scale real time ultrasonograhic examinations were performed using PHILIPS-HD6 ultrasound system. The subjects were scanned with a moderately distended bladder in supine position. The trasducer was placed on the skin surface after applying the coupling agents. All measurements of fetal femur length were made using electronic calipers The gestational age was calculated from the first day of last menstrual period, and corrected for cycle length, i.e. corresponding number of days were added or subtracted according menstrual cycle length shorter or longer than 28 days respectively. The fetal age was confirmed by either first trimester or early second trimester sonographic scans. The age estimation in first trimester was based on crown-rump length measurements and in second trimester was determined with the use of bipariatal diameter (BPD) head circumference and abdominal circumference.

The mean values of femur length in $\mathrm{cm}$. along with respective standered deviation (sd) were computed for each gestational age from 15-40 weeks. The correlation and regression analysis has been carried out to quantify the relationship between gestational age in weeks and femur length in $\mathrm{cm}$.

\section{RESULT}

It is observed that the diphyseal length of femur is increased from 18 weeks to 40 weeks of gestation. The diphyseal length of femur in fetuses of subjects from 18 to 40 weeks of gestation is plotted and it is observed that there is linear relationship between the diphyseal length of femur (in $\mathrm{cm}$ ) and gestation age in weeks (Figure-1). It is observed from 95\% confidence interval that gestational age can be accurately predicted from diphyseal length measurements of femur.

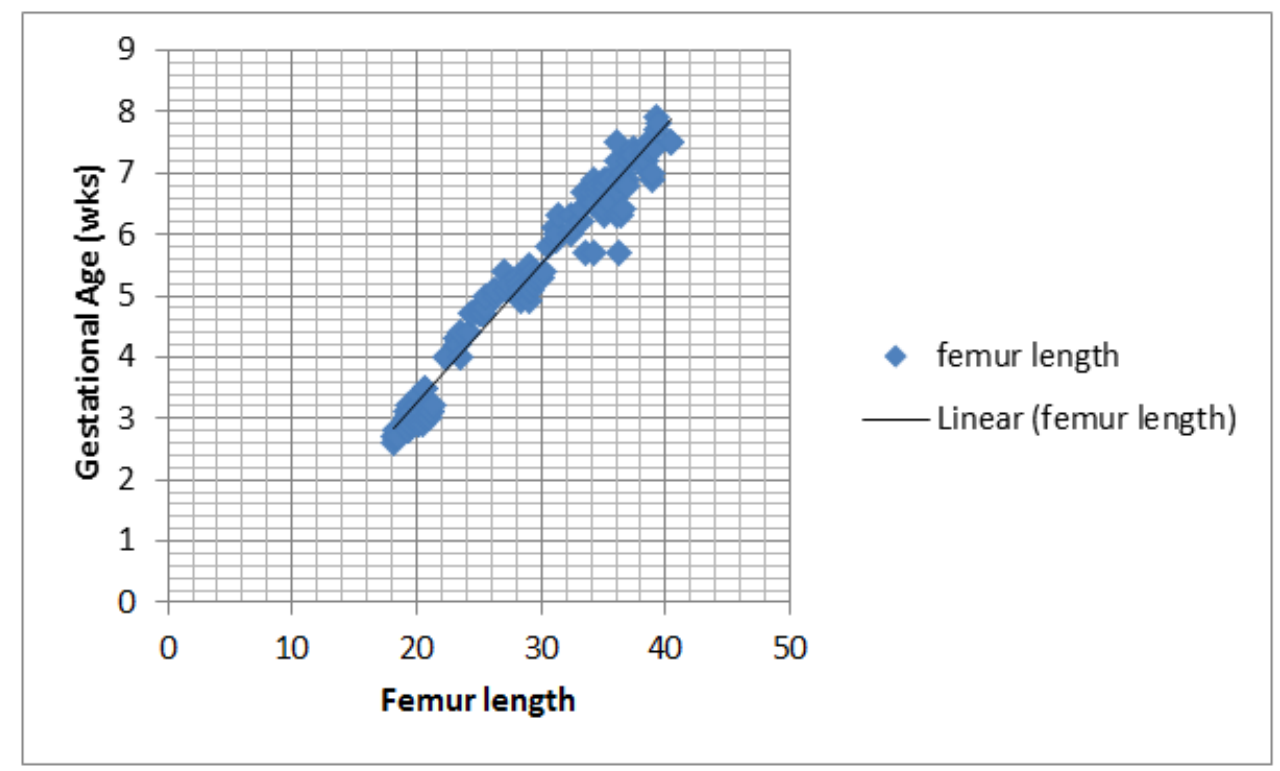

Fig-1: It shows graph plotted with gestational age in weeks against fetal femur length in $\mathrm{cm}$ 
From Table-1 it can be seen that, a significant and positive relationship was observed between gestational age and diphyseal length of femur $(r=0.9827, p<0.05)$ at $5 \%$ level of significance. It means that the gestational age and the diphyseal length of femur are dependent on each other. The diphyseal length of femur (in $\mathrm{cm}$ ) increases with increasing gestational age (in weeks).
The correlation coefficient between the gestational age and diphyseal length of femur is 0.9827 and $\mathrm{t}$-value is 64.55 . Therefore there is positive correlation is observed between the gestational age and the diphyseal length of femur this shows that diphyeal length of the femur is a better predictor of gestational age.

Table-1: Correlation coefficient between the gestational age (in weeks) with the diphyseal length of femur (in cm) $\mathbf{n}=\mathbf{1 5 0}$

\begin{tabular}{|l|l|l|l|}
\hline \multirow{2}{*}{ Length in cm } & \multicolumn{4}{|l|}{ Correlation coefficient between } \\
\cline { 2 - 4 } & Correlation coefficient $\mathbf{~ r ~})$ & t-value & p-value \\
\hline Femur length & 0.9827 & 64.55 & 0.000 \\
\hline
\end{tabular}

Regression analysis of relationship between the gestational age and diphyseal length of femur-The simple linear regression analysis was applied to assess the dependant variable, Gestational age by another independent variable Femur length in total 150 cases as shown in Table-2. The regression coefficient is 6.334 . The regression coefficient for femur length is 4.291 . The data shows that the diphyseal length of femur is directly related to the gestational age .With simple linear regression modeling, equation is derived in the form of $y=a+b 1 * 1$
Where $y$ is dependant variable, $a$ is the regression coefficient constant, b1 is regression coefficient for independent variable and $* 1$ is the independent variable itself i.e.GA $=a+b 1[F L]$

Where GA is gestational age in weeks and FL is the diphyseal length of femur in $\mathrm{cm}$. Therefore

$\mathrm{GA}=6.334+4.291[\mathrm{FL}]$

So by this formula the gestational age can be estimated.

Table-2: Simple linear regression of the gestational age by the diphyseal length of femur (in cm) in total samples

\begin{tabular}{|l|l|l|l|l|l|l|}
\hline $\begin{array}{l}\text { Independent } \\
\text { variable }\end{array}$ & Regression coefficient & $\begin{array}{l}\text { SE of Regression } \\
\text { coefficient }\end{array}$ & t-value & P -value & F-value & $\begin{array}{l}\text { Regression } \\
\text { Equation }\end{array}$ \\
\hline Constant & 6.334 & 0.3653 & 17.33 & 0.00 & 4167.2 & $\begin{array}{l}\text { GA }= \\
6.33+4.29[\mathrm{FL}]\end{array}$ \\
\hline Femur length & 4.291 & 0.0664 & 64.55 & 0.00 & & 6.35 \\
\hline
\end{tabular}

\section{DISCUSSION}

In the present study we have assessed the relationship of sonographic measurement of femur length in cm with gestational age in weeks and growth pattern of femur with advancing gestational age. The study showed that the diphyseal lengh of femur increases steadily with increasing gestational age in a linear fashion. The result of present study similar with the observation made in previous studies. Hadlock F.P et al., [5] studied the relation between fetal femur length and gestational age by cross sectional analysis of 338 normal feuses (12-40 weeks) using real time sonography. Regression analysis of gestational age /femur length relation was carried out on each group using the linear, linear quadratic and linear cubic models. The linear quadratic function was the optimal model for predicting menstrual age from femur length. Zorzoli A et al., [6] measured the fetal femur length in 296 pregnant women at 64-108 days of gestational age, using vaginal sonography. Limb measurements correlated significantly with gestational age, the best description being achieved by a linear regression for all segments. Multiple regression showed that each limb bone correlated independently with both GA and BPD, $\mathrm{FL}=8.26+0.0669 \mathrm{GA}+0.537 \mathrm{BPD}$ where GA in days, FL and BPD are in $\mathrm{mm}$. Exacoustos $\mathrm{C}$ et al., [7] studied the growth patterns of fetal limbs, measurements of femur, made by ultrasound and related it to gestational age in 2317 normal singleton pregnancies at 13 to 40 weeks of gestation. The regression analysis was used to establish relationships between long bone length and gestational age. The second degree polynomial equation turned out to be the best model describing the relationship between femur length and gestational age.

Salomon L.J et al., [8] constructed new reference charts and equations for fetal FL, Bipariatal diameter, Head circumference and abdominal circumference using large sample (19647) of fetuses examined at 15-40 weeks of gestation. Raw data were fitted satisfactorily with a cubic polynomial model for each biometric parameter as follows $\mathrm{FL}=$ $27.085+2.92223 * \mathrm{GA}+0.0148 * \mathrm{GA} 2-0.0006 * \quad \mathrm{GA} 3$ $(\mathrm{R} 2=96.33)$ where $\mathrm{FL}$ is in $\mathrm{mm}$ and $\mathrm{GA}$ is in weeks. Monalisa $\mathrm{R}$ et al., [9] concluded that sonographic examination was effective to assess the accurate femur length parameter for different weeks of gestation.

In the present study the simple linear regression equation derived for the calculation of gestational age using diphyseal length of femur is-

$\mathrm{GA}=6.334+4.291[\mathrm{FL}]$ 
Where GA is gestational age in weeks and FL is the diphyseal length of femur in $\mathrm{cm}$.

The present study is a cross sectional study design, which is made up of observations on different individuals. If we take the serial measurements on same patients throughout gestation, it may provide clear understanding of growth pattern. Accuracy of measurements of femur depends on making a perpendicular scan of the long bone and care should be taken in acquisition and interpretation of the images to prevent spurious measurements. The parameter for long bone length may vary among different population groups. Population specific normograms may be derived from large sample sizes. The long bone growth curves may be different for different population groups.

\section{CONCLUSION}

The relationship between the diphyseal length of femur and the gestaional age is linear and direct. Measurements of diphyseal lengths of femur can be an important parameter for estimating gestational age along with other parameters especially from 18-40 weeks of gestation. The diphyseal length increases with increasing gestational age. Normograms of diphyseal lengths of femur can be consructed by the formula derived by this study for this particular geographical area. The formula derived can be used to determine wheather a given diphyseal lengh of femur is normal or abnormal for a particular gestational age. Sonographic measurements of diphyseal lengths of femur helps in the detection of fetal growth abnormalities that can affect the outcome of pregnancy. Determining diphyseal lenghs of femur may be helpful in the diagnosis of abnormalities; shortened and abnormal long bones are seen in skeletol dysplasias and intrauterine growth retardations.

\section{REFERENCES}

1. Johnson RB, Gregory KD, Niebyl JR. Preconception and prenatal carepart of continuum. In: Gabbe SG, Niebyl JR, Simpson JL, editors.
Obstetrics Normal and problem pregnancies. $5^{\text {th }}$ ed.philadelphia; Churchil Livingstone Elsevier; 2007, 111-137.

2. Dubil EA, Magann EF. Amniotic fluid as a vital sign for fetal wellbeing. Australasian journal of ultrasound in medicine. 2013 May;16(2):62-70.

3. Cunnigham FG, Leveno KJ, Bloom SL, Hauth JC, Rouse DJ, Spong CV. Fetal imaging in Williams Obstetrics. 23th ed. USA The Mcgraw Hill Companies; 2010; 349-371.

4. Rice KJ, Ballas J, Lai E, Hartney C, Jones MC, Pretorius DH. Diagnosis of fetal limb abnormalities before 15 weeks: cause for concern. Journal of Ultrasound in Medicine. 2011 Jul;30(7):1009-19.

5. Hadlock FP, Harrist RB, Deter RL, Park SK. Fetal femur length as a predictor of menstrual age: sonographically measured. American Journal of Roentgenology. 1982 May 1;138(5):875-8.

6. Zorzoli A, Kustermann A, Caravelli E, Corso FE, Fogliani R, Aimi G, Nicolini U. Measurements of fetal limb bones in early pregnancy. Ultrasound in Obstetrics and Gynecology: The Official Journal of the International Society of Ultrasound in Obstetrics and Gynecology. 1994 Jan 1;4(1):2933.

7. Exacoustos C, Rosati P, Rizzo G, Arduini D. Ultrasound measurements of fetal limb bones. Ultrasound in Obstetrics and Gynecology: The Official Journal of the International Society of Ultrasound in Obstetrics and Gynecology. 1991 Sep 1;1(5):325-30.

8. Salomon LJ, Duyme M, Crequat J, Brodaty G, Talmant C, Fries N, Althuser M. French fetal biometry: reference equations and comparison with other charts. Ultrasound in Obstetrics and Gynecology. 2006 Aug;28(2):193-8.

9. Roy M, Gajbe UL, Singh BR, Thute P. Morphometric measurement of fetal femur length for the prediction of gestational age in the IInd and IIIrd trimester of pregnancy by ultrasonography. Journal of Datta Meghe Institute of Medical Sciences University. 2017 Jul 1;12(3):187. 